\title{
Estructura territorial del turismo en el corredor Tijuana-Rosarito-Ensenada
}

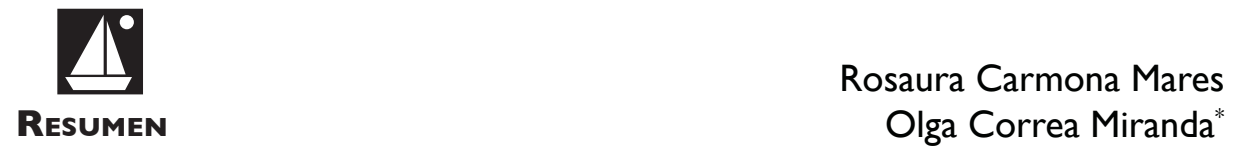

Este trabajo tiene como objetivo central revelar la estructura territorial de la actividad turística en el corredor turístico TijuanaRosarito-Ensenada (CTRE), cuya dinámica ha estado siempre ligada a la economía estadounidense. Durante su proceso de consolidación urbana, el turismo ha sido un factor determinante que se refleja en su estructura territorial. Hay tres secciones básicas en el trabajo: en la primera se refieren los antecedentes históricos de la actividad turística en el CTRE; en la segunda se explica la estructura territorial del turismo; $y$ en la parte final se exponen algunas consideraciones que apuntan a predecir un crecimiento del turismo del CTRE en el futuro mediato.

Palabras | Estructura territorial, corredor turístico, Tijuana-Rosarito-Ensenada. CLAVE

*Universidad Nacional Autónoma de México, Facultad de Filosofía y Letras, Instituto de Geografía / roswita_I @@yahoo.com.mx / olgacorream@yahoo.com 


\section{Introducción}

El turismo es una actividad humana compleja que depende directamente de espacios concretos para su funcionamiento y que mantiene una relación muy estrecha con el territorio; involucra un conjunto de relaciones, influencias, motivaciones, deseos y representaciones; eleva el consumo, desencadena y fomenta diversos servicios y nuevas actividades. Determina la transformación espacial inducida por los equipamientos de ocio, modifica los patrones de ocupación y servicios urbanos; eleva el valor del suelo y genera "cierto patrón de ordenamiento del territorio" tanto en sus dimensiones macrorregionales (internacional-nacional) como en las microrregionales (local-urbano); transforma el paisaje existente y origina nuevas formas de estructuración espacial. En suma, se trata de una actividad que implica una fuerte estructuración y reestructuración del espacio, lo que provoca que el territorio se configure para satisfacer la demanda turística (Coriolano, 1998; Hiernaux, 1989; Vera et al., 1997).

Así, la estructura territorial del turismo es el reflejo de las múltiples relaciones que se establecen entre los recursos turísticos, los actores humanos y los factores técnicos, los cuales son agentes fundamentales en las formas de articulación del turismo en el espacio.

\section{Ubicación y delimitación del CTRE}

En el extremo noroccidental del estado de Baja California se emplaza el corredor Tijuana-Rosarito-Ensenada (CTRE); se trata de una superficie alargada que se extiende en forma continua a lo largo de 140 kilómetros de litoral, desde el fraccionamiento Playas de Tijuana hasta la región de Punta Banda-La Bufadora, Ensenada; tiene un ancho de aproximadamente dos kilómetros tierra adentro que, en algunas zonas, se prolonga hasta los diez kilómetros y abarca una superficie de $613.15 \mathrm{~km}^{2}$. Comprende la porción costera de los municipios de Tijuana, Playas de Rosarito y parte de Ensenada y le corresponde una porción marina con un ancho aproximado de seis kilómetros (tres millas náuticas) con respecto al litoral.Así, de los 140 kilómetros de costa, I 4 pertenecen a Tijuana, 45 a Playas de Rosarito y el resto a Ensenada (Gobierno del Estado de Baja California, 1995; Bringas, 1999; Cocotren, 200 I; mapa I). 


\section{Antecedentes}

Durante varios decenios del siglo $x x$, el desarrollo del CTRE estuvo sujeto a los vaivenes de la economía estadounidense; en esta relación de dependencia, el turismo ha sido uno de los ejes principales de la dinámica económica y social de la región (Bringas, 1999; Gasca, 2000).

El auge económico de la zona se desencadenó durante el período de la Ley Seca en Estados Unidos (1920-1933), debido a la presencia de negocios ligados a la diversión en Tijuana y Ensenada; la gente no visitaba estas ciudades por sus atractivos naturales, sino por la existencia de negocios en donde se expendían bienes y se ofrecían servicios prohibidos en Estados Unidos: casinos, juegos de azar, apuestas y carreras de caballos, venta de alcohol, etc. (Bringas, 1999; INEGI, 2000).

En 1935 se construyó la carretera libre Tijuana-Ensenada, lo que logró una mayor comunicación e integración de la economía regional; en los años siguientes, estas ciudades cobraron una gran importancia turística, por lo que el gobierno federal apoyó su crecimiento por medio de infraestructura vial. En la década de los sesenta se concluyó la carretera de cuota número I (escénica Tijuana-Ensenada), que posibilitó un mayor poblamiento e integración del corredor al conectar los diferentes poblados situados a lo largo de la costa, con lo que se "descubre" el potencial turístico regional, lo cual influyó en el cambio de uso del suelo. También se abrieron nuevas rutas aéreas como las de México-Tijuana y Acapulco-Tijuana, que enlazaron a la zona con el resto del país (Bringas, 1993; Jiménez, I 993; Gasca, 2000; Ceballos, 200I).

Con las nuevas carreteras y cambios en el uso de suelo,la actividad turística en el corredor llegó a convertirse en un elemento cardinal para el crecimiento económico; se desencadenaron obras de infraestructura en materia de dotación de servicios; lo cual permitió la expansión de la ciudad de Tijuana hacia el sur y,junto con ello, en 1970 surgieron y se extendieron algunos campos turísticos (Bringas, 1995).

Fue en los ochenta cuando - a causa de la dotación de la infraestructura de los centros turísticos por parte de prestadores de servicios y no del gobierno del estado- se crearon complejos turísticos alrededor de Rosarito y esta localidad se convirtió en un centro urbano de primer orden, pero también surgieron las inmobiliarias y comenzó la especulación del suelo costero (Bringas, 1995). 
En el último decenio del siglo pasado, el turismo reafirmó su papel central en la economía de Baja California, por lo que el gobierno estatal promovió intensamente la atracción de turistas extranjeros; una muestra clara de ello es la elaboración del Programa Regional de Desarrollo Urbano, Turístico y Ecológico del Corredor Costero Tijuana-Ensenada. Por otro lado, la localidad de Rosarito, perteneciente al municipio de Tijuana hasta 1995, fue constituida como la cabecera de un nuevo ayuntamiento gracias al crecimiento físico de su infraestructura turística y a su autosuficiencia económica derivada, en gran parte, de los ingresos generados por la actividad turística (Propin, Sánchez y López, 1997; Bringas, 1995) véase mapa I.

En la actualidad, un sector de la ciudad de Tijuana, junto con Rosarito y Ensenada, han mostrado un crecimiento extraordinario evidente incluso en el ámbito turístico nacional; su corredor costero, perteneciente a la "Costa Dorada" de Baja California, recibe al menos 25 millones de personas al año procedentes, en su mayoría, de Estados Unidos (Propin, Sánchez y López, I997; Cotuco, 2002).

\section{Estructura territorial del CTRE}

Vías de comunicación terrestre

La principal vía de comunicación terrestre de la península de Baja California es la carretera transpeninsular, y asociada a ésta se encuentra la escénica de cuota Tijuana-Ensenada, una de las vías de mayor relevancia del CTRE; inicia en el extremo noroeste de Tijuana, cruza de norte a sur el municipio de Playas de Rosarito y finaliza en el norte de la ciudad de Ensenada; su flujo vehicular es predominantemente turístico y es utilizada en forma significativa por los visitantes procedentes de Estados Unidos.

Sin duda, los asentamientos urbanos del corredor tienen un patrón lineal de localización determinado por las dos vialidades antes señaladas. En Playas de Tijuana, la carretera escénica es la única vía de comunicación de primer orden, la cual se extiende por su parte este al centro de la ciudad y los cruces internacionales San Ysidro y Mesa de Otay, y hacia el sur con el municipio de Playas de Rosarito.

La porción de Playas de Rosarito también está articulada linealmente a partir de la carretera número I que, proveniente del norte, cruza la cabecera 


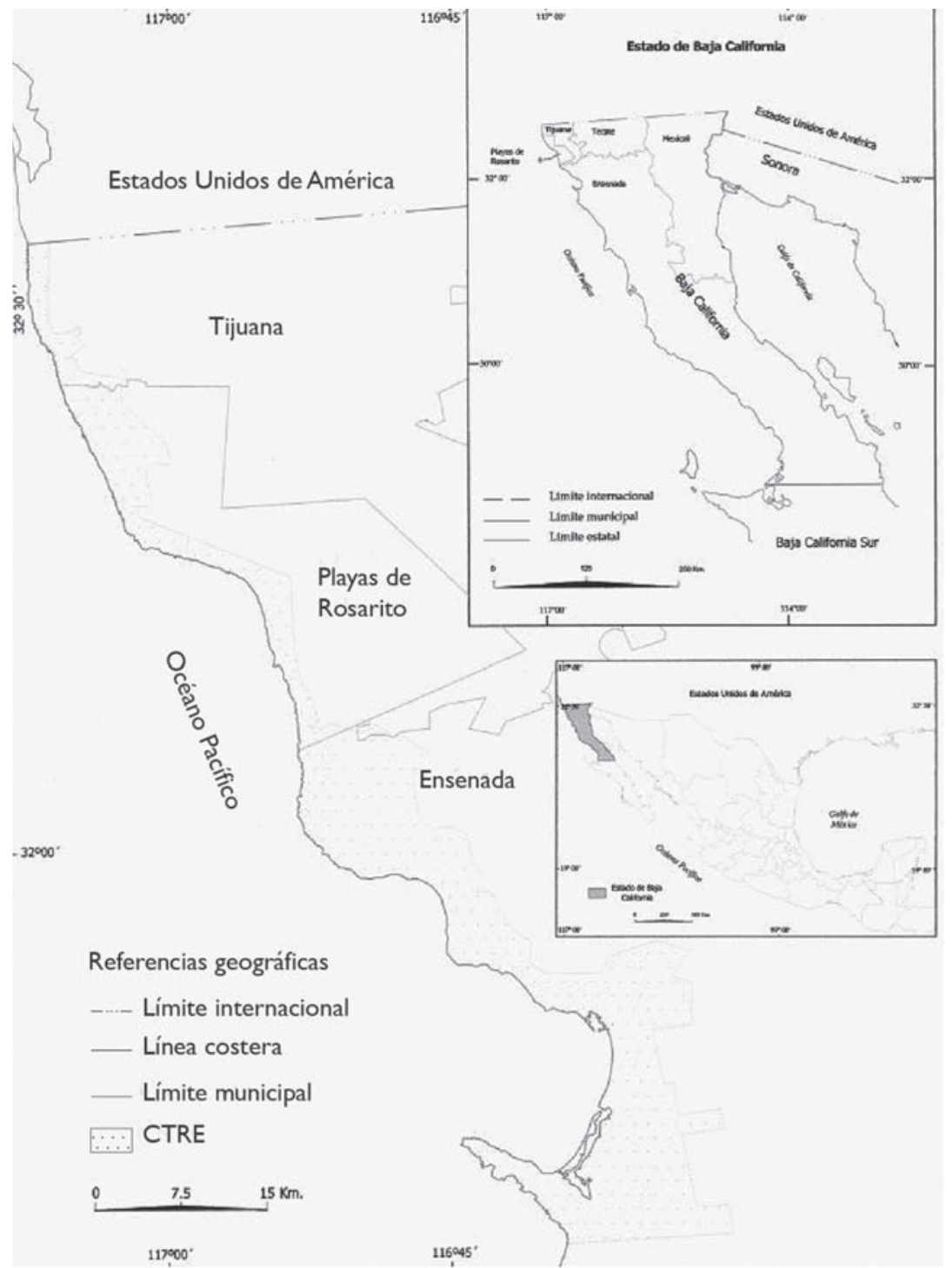


municipal de Playas de Rosarito, y continúa al sur con dirección al poblado La Misión; en su recorrido, une los centros turísticos más importantes del municipio. Sigue su trayectoria a lo largo de la costa hasta el arroyo La Misión e ingresa al municipio de Ensenada.

La situación de las vialidades I y ID cambia al entrar al municipio de Ensenada; si bien se mantienen relativamente paralelas a la costa, la carretera I se aleja de ella, mientras que la autopista ID se mantiene como costera y enlaza diferentes centros turísticos del municipio. Ambas vías se unen en El Sauzal, al norte de la ciudad de Ensenada, y a partir de este punto se convierten en la carretera transpeninsular, principal vía de la ciudad y del sur del municipio, que entronca con el valle del Maneadero, donde inicia la carretera estatal número 23, que se dirige a Punta Banda y a La Bufadora, destinos turísticos tradicionales del estado.

\section{Otras vías de comunicación}

Las vías de comunicación aérea que existen en el corredor no atienden a la demanda de turistas a gran escala; el aeropuerto militar "El Ciprés" se encarga ocasionalmente de algunos vuelos comerciales y privados de pasajeros, en embarcaciones pequeñas.Además, existe una aeropista en Punta Banda en la cual se reciben pequeñas aeronaves turísticas, donde son transportados los socios del club privado La Grulla. Por otro lado, el Aeropuerto Internacional de Tijuana, aunque no se encuentra dentro de los límites físicos del corredor debido a su amplio alcance regional, es utilizado por los turistas que arriban al corredor.

En cuanto a la infraestructura portuaria, cuatro de los puertos de Baja California, Ensenada, El Sauzal, Rosarito e Isla de Cedros tienen instalaciones adecuadas para tráfico de altura y cabotaje; el puerto de San Felipe es sólo de cabotaje. En todos ellos pueden realizarse actividades pesqueras, comerciales y turísticas, particularmente en los ubicados en el CTRE (INEGI, 2000; Cocotren, 200 I; Colef, 2004; mapa 2).

\section{Uso y valor del suelo en el CTRE}

Según la dinámica turística del CTRE, el uso de suelo turístico ha registrado un crecimiento constante basado en la construcción de centros y fraccionamientos turísticos que acaparan los espacios costeros y especulan con el precio 
de los mismos.Así, el turismo influye en el actual tipo de ocupación y costo del suelo del CTRE.

Los asentamientos poblacionales del CTRE siguen un patrón lineal que forma un continuo en el que se han consolidado los centros urbanos más importantes: Playas de Tijuana, Playas de Rosarito, El Sauzal, Ensenada y localidades de menor jerarquía como El Descanso, Popotla, El Morro, Primo Tapia, La Misión, Santa Anita, Ejido Chapultepec, Ejido Rodolfo Sánchez Taboada y Ejido Esteban Cantú; juntos conforman el sistema regional de ciudades del corredor (Cocotren, 200I).

El acelerado crecimiento demográfico y la dinámica económica generados en torno a estos ejes carreteros han fomentado la conurbación del sector Playas de Tijuana con el centro de población Playas de Rosarito, fenómeno que repercute directamente en el uso del suelo que se presenta en esta zona. El sector de Playas de Tijuana no está integrado con el centro de la ciudad, el nivel económico de los residentes es medio y medio alto, y no se ha dado una distribución de usos de suelo equilibrada, capaz de satisfacer las necesidades básicas de su población. El uso que predomina es el habitacional, que incluye los fraccionamientos y condominios turísticos.

El uso comercial y de servicios se encuentra a lo largo de las principales avenidas; el turístico, en la sección costa y longitudinal a la carretera escénica Tijuana-Ensenada, en las localidades de La Joya, Punta Bandera, Real del Mar y San Antonio del Mar; también existe un uso de suelo rústico en pequeños segmentos al este de la carretera.

La concentración de asentamientos urbanos de Playas de Rosarito se da en el norte del municipio en el continuo urbano que forma conTijuana, a lo largo de la costa. Esta parte del corredor es en su mayoría habitacional y contiene grandes zonas de baldíos, lo que revela una falta de consolidación urbana. El uso turístico se ubica en el norte de la ciudad de Rosarito, en El Oasis, Baja del Mar y Santa Mónica; hacia el sur, a lo largo de la costa en Popotla, El Morro y Primo Tapia en donde predominan condominios turísticos. El suelo dedicado a los comercios y servicios se localiza a lo largo de la carretera libre Tijuana-Ensenada, donde se encuentran los principales hoteles y restaurantes de la ciudad.

En la ciudad de Ensenada, el uso habitacional es el más extendido, pero en el centro histórico y en el puerto se concentran comercios y servicios enfocados en su mayoría a la actividad turística. Hacia el sur de la ciudad, en el ex Ejido 
Chapultepec, prevalece el uso habitacional y resalta el de equipamiento, con la presencia del aeropuerto de Ensenada. Hacia el sur del estero Punta Banda en La Joya, se localiza un área habitacional constituida en su mayor parte por fraccionamientos turísticos. En Punta Banda y en la barra que separa el estero de la bahía es dominante el uso de suelo turístico debido a sus particulares atractivos naturales.

El valor por metro cuadrado del suelo en los espacios turísticos, centros de ciudades y las franjas costeras es alto y en su determinación intervienen propietarios, instituciones gubernamentales, inmobiliarias, constructoras y los usuarios (Verduzco, Bringas y Valenzuela, 1995).

En el CTRE, un espacio donde el turismo es el eje principal de desarrollo articulado por los centros urbanos y costeros, las zonas de máximo valor tienen un costo que oscila entre los 91 y 217 dólares por metro cuadrado y corresponden a I) los centros turísticos de primer orden de Playas de Tijuana, Playas de Rosarito y el centro y el puerto de la ciudad de Ensenada, que son los que tienen la mejor localización, accesibilidad, presencia de infraestructura y una intensa actividad comercial y turística; y 2) desarrollos turísticos de menor jerarquía que, conectados a los núcleos ya señalados por las carreteras costeras libre y de cuota Tijuana-Ensenada, han logrado tener una infraestructura mínima a partir de sus atractivos, fraccionamientos, condominios y fideicomisos turísticos, como los de Punta Bandera, San Antonio del Mar y Baja Malibú, en Tijuana; El Oasis, Baja del Mar y Santa Mónica, al norte del municipio de Playas de Rosarito;y, hacia el sur, dos continuos, uno de Popotla a Cantamar y el otro de Misión Viejo a la playa La Misión; en el municipio de Ensenada se encuentran La Salina, Bajamar, la playa de Cíbolas del Mar y El Sauzal.

Las áreas colindantes con las de máximo valor alcanzan entre 37 y 90 dólares por metro cuadrado, y se trata de sitios: I) que están estrechamente vinculados con lugares costeros que median entre los centros de máximo valor y con potencial de desarrollo turístico; 2) que por estar emplazados a lo largo de las principales vías de comunicación, registran una dinámica comercial intensa, como las franjas aledañas a la carretera libreTijuana-Ensenada;3) que cuentan con el grado más elevado de urbanización dentro de los centros de población, como es el caso de Playas de Rosarito y Ensenada;y 4) que están relacionados directamente con la actividad turística de los municipios de Playas de Rosarito y Ensenada (Punta Banda), donde los atractivos turísticos naturales únicos influyen de manera preponderante en la determinación del costo del suelo. 


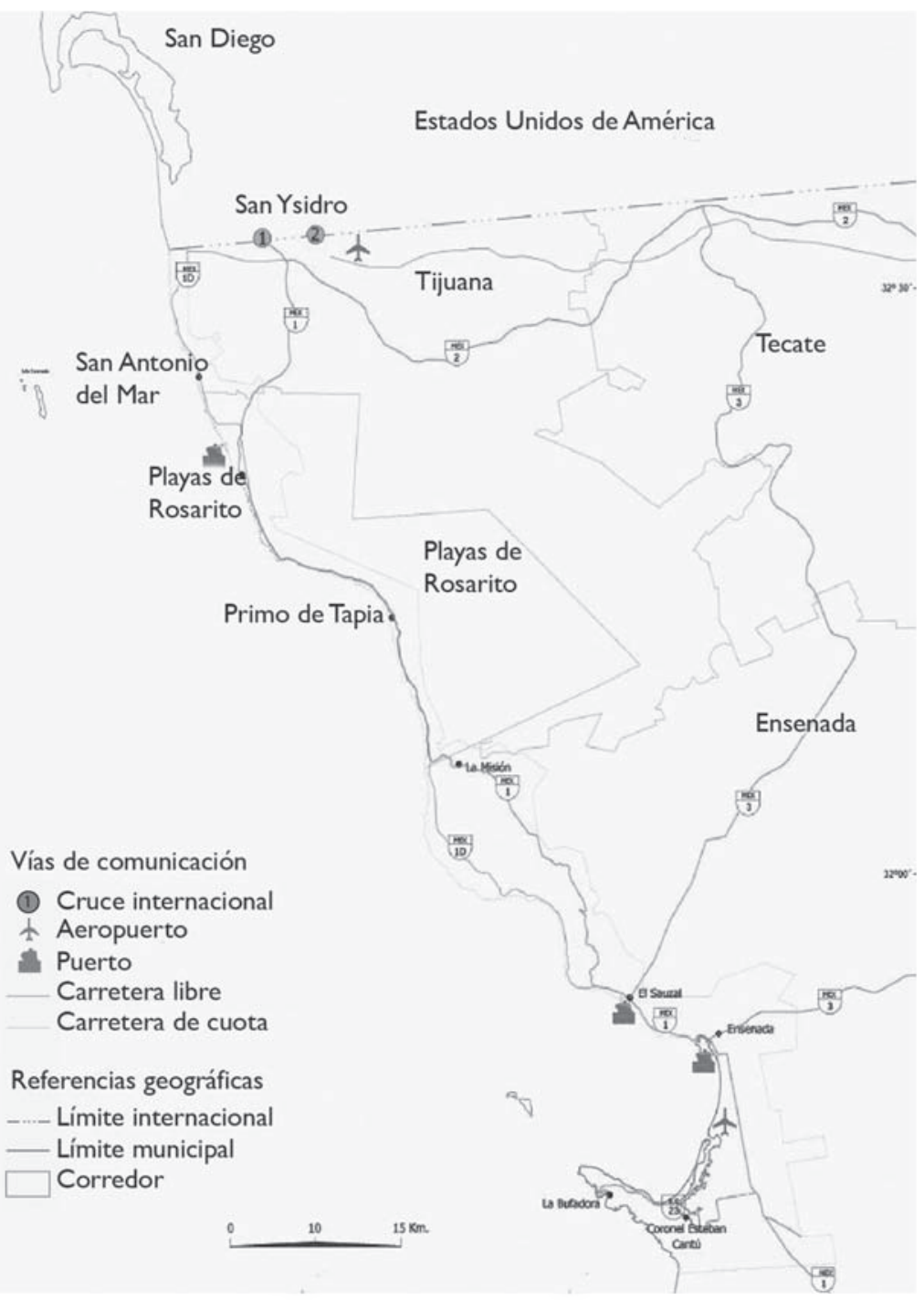

Fuente: Elaboración propia con base en Secretaría de Comunicaciones y Transportes, 1999.

Figura 2.Vias de comunicación del Corredor Tijuana-Rosarito-Ensenada (2006) 
Así, en la mayoría de los casos, el valor catastral del suelo en el CTRE expresa la influencia de diversos agentes como el uso de suelo rural o urbano, la ubicación, la accesibilidad, los paisajes y la presencia o ausencia de infraestructura. Además, el cambio de uso de suelo de rural a urbano promueve un aumento en su valor, por la presión de desarrolladores estatales o privados, lo que a su vez lo incrementa en las áreas circunvecinas, derivado de la especulación que se da respecto a estos predios. Esta situación también se observa en la actividad turística, en la cual se vive una intensa competencia por los mejores lugares, lo que tiene como consecuencia la segregación extrema del espacio por la disposición y utilización del suelo (Verduzco, Bringas y Valenzuela, I 995; López, 200I;Texiera, 1996).

\section{Recursos turísticos naturales y culturales del CTRE}

Los factores espaciales de atracción en el CTRE han evolucionado conforme a las demandas de los viajeros que arriban a esta región; en un primer momento se trataba de turistas que buscaban atractivos que no estaban relacionados en forma directa con el medio natural; ahora, la oferta se ha diversificado y se basa en atractivos naturales y culturales, así como en otras opciones que se encuentran en los alrededores (mapa 3).

\section{Recursos naturales}

Las actividades turísticas del CTRE tienen como eje rector sus recursos costeros y marinos; las playas son uno de los principales, $y$, aunque no todas ofrecen las condiciones ideales de clima y rasgos del oleaje para su uso como balneario, este litoral es muy buscado por los viajeros provenientes de latitudes más altas, pues, entre otros aspectos, el clima es más benigno.

Según las características de las playas, las actividades realizadas pueden ir desde el descanso hasta la práctica de deportes extremos. La mayoría de las playas del CTRE se encuentran rodeadas de acantilados rocosos, de modo que su paisaje es una combinación de montaña, desierto y cuerpos de agua costeros. A lo anterior se suma una vegetación y una fauna singulares, lo que evidencia un equilibrio estético ampliamente usado para publicitar esta región. Los asentamientos turísticos se han incrementado en los territorios que poseen paisajes 
excepcionales, en donde es bastante redituable invertir en fraccionamientos de lujo para turistas, sobre todo extranjeros. Los paisajes del CTRE son apreciados de manera especial en los miradores ubicados en puntos estratégicos, sobre todo en acantilados.

La pesca deportiva es un importante atractivo para los paseantes; se practica en Playas de Tijuana, Playas de Rosarito, La Salina, Ensenada y La Bufadora, que son puertos de altura con marinas de primer nivel o incluso embarcaderos rústicos con rampas de boteo y servicios básicos; además, se organizan torneos anuales para impulsarla.

Por otro lado, el avistamiento de fauna en su medio natural siempre se ha promocionado como un elemento que conquista a los visitantes. En las costas bajacalifornianas es posible observar una gran diversidad de especies, algunas de ellas endémicas, pero el interés turístico se centra en los mamíferos marinos.

\section{Recursos culturales}

Éstos se concentran en las zonas urbanas del CTRE y la mayoría son localidades recientes, por lo que hay pocos edificios históricos que se promueven con fines turísticos; más bien lo que se publicita son equipamientos turísticos específicos y culturales: plazas de toros, instalaciones deportivas, marinas, infraestructura hotelera, servicios de lujo (como los Spas), galerías de arte, artesanías, su reconocida vida nocturna y su gastronomía, además de múltiples actividades programadas.

En los principales centros urbanos del corredor se han implantado paseos peatonales turísticos para optimizar el tiempo de estadía de los turistas en un lugar, como los que se ofertan en Playas de Rosarito y Ensenada; se visitan los puntos más interesantes de la zona y la duración es de dos o tres horas; eventualmente, pueden consumir todo el día, según los intereses de cada turista.

\section{Infraestructura ligada al turismo en el CTRE}

Como ya se mencionó, los factores técnicos son los principales indicadores de la consolidación y de la transición de un espacio turístico, y comprenden la infraestructura y los servicios del destino, que facilitan la estadía y el movimiento de personas así como la circulación de bienes e información entre las zonas emisoras y receptoras (Lozato, 1990;Vera et al., 1997).

En la interpretación de los espacios turísticos, contemplar la distribución de la infraestructura de alojamiento es fundamental, pues en torno a ella se 
Estructura territorial del turismo en el corredor Tijuana-Rosarito-Ensenada

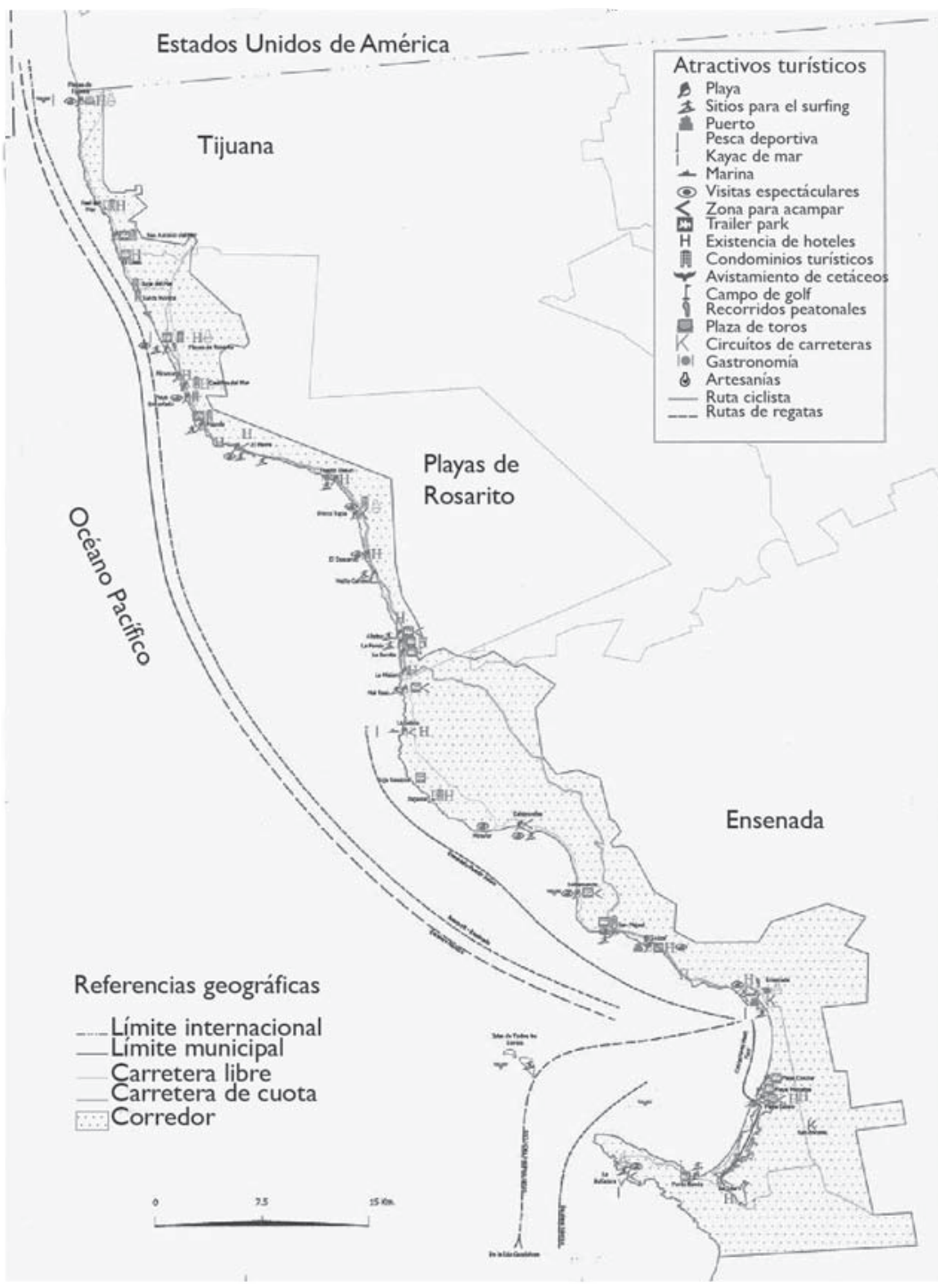

Fuente: Elaboración propia con base en INEGI, 200I.

Figura 3.Atractivos turísticos del Corredor Tijuana-Rosarito-Ensenada 
ofertan otros servicios como los de alimentos y diversión; junto con estos complejos, la presencia de riquezas naturales o culturales, que funcionan como un factor de localización (Aznar, 2003; López, 2001).

Entre el 2000 y el 2004, la oferta de unidades de hospedaje en el CTRE presentó pequeñas fluctuaciones, con tendencia a la baja, debido al incremento de llegadas de visitantes a otros tipos de alojamiento como tráiler parks, campamentos, condominios turísticos y fraccionamientos de lujo destinados a segundas residencias de extranjeros, de modo que se dificulta mantener un registro preciso de la cantidad de turistas recibida, $y$, en este sentido, las cifras están subestimadas.

Al inicio de 2006, fueron registradas en el CTRE 6050 habitaciones, de las cuales $8.1 \%$ se encuentran en el sector de Playas de Tijuana (en 14 establecimientos), $37.8 \%$ (35 establecimientos) en la sección de Playas de Rosarito y 54. I\% (83 establecimientos) en el municipio de Ensenada; esta distribución porcentual evidencia la oferta y la demanda en cada sector del corredor.

Casi todas las unidades de hospedaje son de cuatro y tres estrellas; sólo ocho de los 132 establecimientos del corredor son de cinco estrellas, lo que denota el predominio del turista con una menor capacidad adquisitiva que el de otros centros litorales mexicanos de lujo como Los Cabos o la Riviera Maya, donde hay varias unidades de clase especial y gran turismo; esto se debe, en parte, a que los turistas con más alto poder económico no son registrados en hoteles, pues en su gran mayoría ocupa residencias en fraccionamientos de lujo; se trata principalmente de extranjeros que compran bajo el régimen de fideicomiso. Otra forma de alojamiento para turistas extranjeros con un rango de mediano a alto poder adquisitivo son las residencias de lujo que también son adquiridas como fideicomisos; este tipo de desarrollos inmobiliarios abundan en el sector de Playas de Rosarito (30 de los 40 existentes en el CTRE), y ofrecen ventajas en cuanto a la dotación de infraestructura y servicios, además de las mejores vistas y acceso a las playas (Cocotren, 200I).

En el CTRE hay 33 establecimientos que no entran en la categorización en estrellas de la Secretaría de Turismo: se trata de moteles, cabañas, villas y tiempos compartidos, modalidades que se concentran en Ensenada, donde existen 24 sitios que emplazan estas formas de alojamiento, $y$, en menor medida, en Tijuana y Rosarito, donde en conjunto suman nueve lugares. A éstos se les agregan los trailer parks, que dan cabida a casas rodantes o vehículos 
recreativos; en el corredor hay 64, de los cuales 33 se localizan en Playas de Rosarito, 30 en Ensenada y uno en Tijuana; en algunos casos, éstos funcionan como segundas residencias por el tiempo de arrendamiento, que puede extenderse hasta diez años (Cocotren, 200 I; Colef, 2004).

Asimismo, en el CTRE hay 16 terrenos habilitados para acampar, ubicados en áreas cercanas a las playas y a los acantilados o en zonas de conservación que ofrecen los mejores paisajes y escenarios costeros donde la vista al mar es el elemento más valorado; en Playas de Rosarito se encuentran seis sitios con 197 plazas, y en Ensenada, sobre todo en Punta Banda, diez lugares con I 605 plazas (Cocotren, 200I).

En el sector Playas de Tijuana, la diversidad de hospedaje es limitada; al norte se localizan unidades con categoría de tres estrellas y menos, y al sur, la de mayor lujo. Playas de Rosarito, por su parte, contiene la mayor oferta hotelera de cinco y cuatro estrellas y presenta una especialización hacia los fraccionamientos residenciales de lujo, así como hacia la renta de espacios para tráiler parks. En Ensenada, la oferta hotelera está constituida por establecimientos de categoría menor a dos estrellas y sin categoría; además, en este sector existe el mayor número de espacios para acampar situados en la zona de Punta Banda. En función de las características de alojamiento antes señaladas, se confirma que Playas de Rosarito es el sector de más alto nivel del corredor.

Servicios de alimentos, bebidas y diversión

Los servicios de alimentación juegan un papel principal en la oferta turística del CTRE. La Secretaría de Turismo del Estado (Secture) tiene registrados I38 establecimientos de alimentos con categoría turística, de los cuales $7 \%$ se encuentra en Playas de Tijuana, $46 \%$ en Playas de Rosarito (en su mayoría restaurantes de comida internacional) y $47 \%$ en Ensenada (aglutina dos en la zona centro de la ciudad).

En el CTRE existen 42 establecimientos turísticos asociados con la diversión: bares, cantinas y discotecas; Rosarito tiene 21 de ellos, siendo los más frecuentados por los turistas aquellos que se encuentran en la zona de la playa; en Ensenada, de los 20 negocios registrados, los más concurridos son los que están ubicados en la calle Primera y sus alrededores; por último, en el sector de Playas de Tijuana, sólo hay un bar de categoría turística. 


\section{Conclusiones}

El incremento demográfico y la dinámica económico-turística que se ha generado en torno a los principales ejes carreteros del CTRE han fomentado la conurbación del sector Playas de Tijuana con las áreas más densamente pobladas del de Playas de Rosarito. Este proceso de ocupación costera permite prever que en un futuro mediato se formará un continuo urbano desde Playas de Tijuana hasta Ensenada.

Aunque las segundas residencias son parte indisoluble de la oferta inmobiliaria en espacios turístico-litorales del mundo en general y de México en particular, lo cierto es que en el CTRE han crecido de manera extraordinaria, sobre todo por la facilidad de compra y el acceso que la población radicada en Estados Unidos tiene en la zona. En consecuencia, se han creado extensas áreas de fraccionamientos en fideicomiso, la mayoría de ellos de lujo, separados notablemente de otras áreas habitacionales. En la medida en que esta tendencia se mantenga, en el CTRE se originará un proceso de segregación territorial que hará más evidentes las diferencias sociales, económicas, de servicios e infraestructura.

A pesar de la importancia que el CTRE ha adquirido en el ámbito internacional por su actividad turística, lo cierto es que se ha explotado de forma intensiva el espacio estrictamente de litoral. En este sentido, no se han integrado otro tipo de recursos, ubicados tierra adentro de la península. Esto ha supuesto una carga turística elevada que convendría revisar en un futuro próximo, con la intención de evitar consecuencias negativas al medio ambiente.

\section{Fuentes Consultadas}

Aznar,J. (2003).“Análisis estructural del sector turístico en Almería”. Cuadernos de Turismo, II, 21 -39.

Boullón, R. (1990). Planificación del espacio turístico. México:Trillas.

Bringas, Nora L. (1993). "Uso del suelo y configuración turística del corredor Tijuana-Ensenada”. Urbanización y servicios, vol. I. México: El Colegio de la Frontera Norte (Colef)/Universidad Autónoma de Ciudad Juárez, 69-95.

(1995). "Turismo, municipio y desarrollo regional en la frontera norte: el caso del corredor turístico Tijuana-Ensenada”, en Tonatiuh Guillén 
López y Gerardo M. Ordóñez Barba (coords.). El municipio y el desarrollo social de la frontera norte. México: Colef/Fundación Friedrich Ebert, 93-129.

(1999). Políticas de desarrollo turístico en dos zonas costeras del Pacífico mexicano. Sonora: El Colegio de Sonora.

Ceballos, M. (200I). Encuentro en la frontera: mexicanos y norteamericanos en un espacio común. México: El Colegio de México/Colef/Universidad de Tamaulipas.

Cocotren (200I).Programa de Desarrollo Urbano,Turístico y Ecológico del Corredor Costero Tijuana-Rosarito-Ensenada. Baja California: Secretaría de Asentamientos Humanos y Obras Públicas.

Colef (2004). Programa de Ordenamiento Territorial. Baja California. México: Colef.

Coriolano, L. (1998). De lo local a lo global: el turismo litoral Crearense. São Paulo: Papirus Editora.

Cotuco (Comité de Turismo y Convenciones de Tijuana) (2002). Manual para convenciones y eventos especiales. Tijuana: $\mathrm{H}$.Ayuntamiento de Tijuana.

Gasca, J. (2000). Organización e integración del espacio binacional: el caso de la frontera México-Estados Unidos. Disertación doctoral. México: Facultad de Filosofía y Letras, Universidad Nacional Autónoma de México (UNAM).

Gobierno del Estado de Baja California (1995). Programa Regional de Desarrollo Urbano, Turístico y Ecológico para el Corredor Costero Tijuana-Ensenada. Baja California: Gobierno del Estado.

Hiernaux, D. (1989). “El espacio reticular del turismo en México”. Geografia y Desarrollo, México, 2, 2, 3, 31-39.

INEGI (2000). Tijuana-Ensenada, México: Guía turística urbana.Aguascalientes: Instituto Nacional de Estadística, Geografía e Informática.

(200I). Síntesis de información geográfica del estado de Baja California.Aguascalientes: Instituto Nacional de Estadística, Geografía e Informática.

Jiménez, A. (1993). Turismo: estructura y desarrollo: la estructura funcional del turismo internacional y la política turística de México: desarrollo histórico, 1945-1990. México: McGraw-Hill.

López, A. (200I). Análisis de la organización territorial del turismo de playa en México, 1970-1996. El caso de Los Cabos, B.C.S. Disertación doctoral. México: Facultad de Filosofía y Letras, UNAM. 
Lozato, J. (1990). Geografia del turismo. Barcelona: Masson.

Marchena (1999). "Turismo cultural: el caso de Sevilla”. Cuadernos de Turismo, 4, 33-50.

Propin, E., A. Sánchez y A. López (1997). “Situación geográfico-económica del turismo en el extremo norte de América Latina: el corredor TijuanaRosarito-Ensenada". Revista de Geografia Norte Grande, Santiago de Chile, Universidad Católica de Chile, 24, 257-26I.

Secretaría de Comunicaciones y Transportes (1999) Baja California: mapa de comunicaciones y transportes. Escala I:530 000. México

Texiera,A. (1996). "El turismo y su influencia en la ocupación del espacio litoral”. Turismo y geografia. Reflexiones teóricas y enfoques regionales. São Paulo: Hucitec, I77-183

Vera, J. et al. (1997). Análisis territorial del turismo. Barcelona:Ariel.

Verduzco, Basilio, Nora L. Bringas y Basilia Valenzuela (1995). La ciudad compartida. Desarrollo urbano, comercio y turismo en la región Tijuana-San Diego. Guadalajara: Universidad de Guadalajara/Colef. 
